\author{
References \\ Falkinham JO. Nontuberculous mycobacteria in the environment. Clin Chest Med 2002; 23: 529-551. \\ 2 Griffith DE, Aksamit T, Brown-Elliott BA, et al. An official ATS/IDSA statement: diagnosis, treatment, and \\ prevention of nontuberculous mycobacterial diseases. Am J Respir Crit Care Med 2007; 175: 367-416. \\ 3 Hoefsloot W, Boeree MJ, van Nieuwkoop C, et al. No human transmission of Mycobacterium malmoense in a \\ perfect storm setting. Eur Respir J 2012; 40: 1576-1578. \\ 4 Colombo RE, Hill SC, Claypool RJ, et al. Familial clustering of pulmonary nontuberculous mycobacterial disease. \\ Chest 2010; 137: 629-634. \\ 5 Bryant JM, Grogono DM, Greaves D, et al. Whole-genome sequencing to identify transmission of Mycobacterium \\ abscessus between patients with cystic fibrosis: a retrospective cohort study. Lancet 2013; 381: 1551-1560. \\ 6 Falkinham JO 3rd. Nontuberculous mycobacteria from household plumbing of patients with nontuberculous \\ mycobacteria disease. Emerg Infect Dis 2011; 17: 419-424. \\ 7 Public Health England. Three-year average tuberculosis case reports and rates by Local Authority, England, \\ 2010-2012. London, Dept of Health, 2013. \\ 8 Moore JE, Kruijshaar ME, Ormerod LP, et al. Increasing reports of non-tuberculous mycobacteria in England, \\ Wales and Northern Ireland, 1995-2006. BMC Public Health 2010; 10: 612. \\ 9 Bruijnesteijn van Coppenraet LES, Savelkoul PHM, Buffing N, et al. Amplified fragment length polymorphism \\ analysis of human clinical isolates of Mycobacterium haemophilum from different continents. Clin Microbiol Infect \\ 2009; 15: 924-930. \\ 10 Lichtenstein MR, Takimura Y, Thompson JR. Photochromogenic mycobacterial pulmonary infection in a group of \\ hospitalized patients in Chicago. II. Demographic studies. Am Rev Respir Dis 1965; 91: 592-595.
}

\title{
Early BCG vaccination is unrelated to pulmonary immunity against Mycobacterium tuberculosis in adults
}

\author{
To the Editor:
}

Vaccination with Mycobacterium bovis bacille Calmette-Guérin (BCG) is performed for the prevention of tuberculosis. M. bovis BCG vaccination is among the most commonly applied of all vaccines worldwide [1]. $M$. bovis BCG vaccination efficiently reduces the morbidity and mortality of tuberculosis in children, especially miliary tuberculosis and meningitis [2].

Although recent investigations of Mycobacterium tuberculosis-specific immune responses by interferon- $\gamma$ release assays (IGRAs) provide evidence on the effect of $M$. bovis BCG vaccination on the prevention of primary infection with M. tuberculosis [3-5], it has been suggested that this effect diminishes during adolescence $[2,6]$. Consequently, adults are probably not protected from pulmonary tuberculosis by BCG vaccination.

To date, no study has investigated the impact of M. bovis BCG vaccination performed in childhood on pulmonary immune responses in adults. The objective of this study was to assess the effect of childhood M. bovis BCG vaccination on systemic and pulmonary immune responses to $M$. tuberculosis in healthy adult individuals exposed to patients with acid-fast bacilli (AFB)-positive sputum smear-positive tuberculosis in Germany.

An observational, cross-sectional, multicentre study was conducted by the German Ministry of Education and Research-funded research consortium on "Pulmonary Tuberculosis - Host and Pathogen Determinants of Resistance and Disease Progression (TB or Not TB)". Healthcare workers (HCWs) with 1) ongoing professional contact with patients with AFB sputum smear-positive tuberculosis, 2) a cumulative professional exposure of at least 2 years, and 3) no clinical signs and/or symptoms of active tuberculosis were recruited at 18 German pulmonary medicine centres (centres are listed in the Acknowledgements section).

Furthermore, household contacts (HHCs) without evidence of active tuberculosis were enrolled at three urban municipal healthcare centres (i.e. Frankfurt, Hamburg and Hannover); their enrolment required 1) the absence of clinical signs and/or symptoms of active tuberculosis, and 2) cumulative exposure of $>40 \mathrm{~h}$ to an AFB sputum smear-positive patient with culture-proven pulmonary tuberculosis. Individuals with a history of pulmonary tuberculosis who completed a standard course of tuberculosis treatment $>6$ months before enrolment and did not experience a relapse were recruited in a control group.

Epidemiological, clinical and demographic data, including BCG vaccination status, were captured using an ad hoc standardised questionnaire. An unblinded physician verified M. bovis BCG vaccination by clinical 
examination of a scar or through the subject's vaccination passport. IGRAs were performed on cells collected from the peripheral blood of all subjects by the QuantiFERON Gold In-Tube (QFT; Cellestis Qiagen, Chadstone, Australia) or T-SPOT.TB (ELISPOT; Oxford Immunotec, Oxford, UK) at the attending physician's discretion. The use of two different test systems impaired the overall comparability of the data but reflected clinical practice in the 18 centres involved. Tuberculin skin tests were not regularly performed according to German guidelines [7]. The laboratory staff was blinded to the clinical data, vaccination status and tuberculosis disease status of the participants.

All enrolled contacts were offered a bronchoscopy with bronchoalveolar lavage (BAL), unless bronchoscopy was contraindicated for medical reasons. If the subject agreed, flexible bronchoscopy was performed according to the current German guidelines [8]. The bronchoscope was wedged into a subsegmental bronchus of the middle lobe. BAL was performed with a total volume of 200-250 mL sterile normal saline.

A T-SPOT.TB was performed on BAL mononuclear cells (BALMCs). Interferon- $\gamma$ secretion was evaluated in response to the following mycobacterial antigens: 6-kDa early secretory antigenic target (ESAT-6), 10-kDa culture filtrate protein (CFP-10) and purified protein derivative (PPD; Statens Serum Institutet, Copenhagen, Denmark). The assay was conducted according to the manufacturer's instructions with the exception that 200000 , instead of 250000 , mononuclear cells were plated per well for improving the visibility of the spots.

Qualitative variables were expressed as percentages, whereas mean $\pm \mathrm{SD}$ ) and median (interquartile range (IQR)) were used for parametric and nonparametric quantitative variables, respectively. The Shapiro-Wilk test, Chi-squared test, t-test and Mann-Whitney test were performed as applicable using Stata 11.0 (StataCorp LP, College Station, TX, USA).

Between June 2008 and July 2012, 512 eligible HCWs and HHCs with known BCG vaccination status were identified (263 HCWs, 166 HHCs and 83 with unclassified exposure). The exposure classification was assigned through a questionnaire, but this information was not captured in the initial phase of the study. Of the remaining 505 HIV-uninfected HCWs and HHCs, 322 (63.8\%; 153 HCWs, 113 HHCs and 56 with unclassified exposure) were immunised with M. bovis BCG during childhood, while 183 (36.2\%; 103 HCWs, 53 HHCs and 27 with unclassified exposure) were not. In $22(4.4 \%)$ persons (12 HCWs and 10 with unclassified exposure), QFT results were unavailable. In 483 contacts with known blood IGRA status, 170 (35.2\%; 92 HCWs, 55 HHCs and 23 with unclassified exposure) had a positive test result. To assess pulmonary immune responses, 88 (17.4\%) out of 505 individuals (31 unvaccinated and 57 M. bovis BCG vaccinated) underwent bronchoscopy with subsequent BALMC IGRA. Of these, 26 (29.5\%; 17 HCWs, four HHCs and five with unclassified exposure) had a positive test result, with $10(32 \%)$ in the unvaccinated and $16(28 \%)$ in the vaccinated group.

M. tuberculosis-specific IGRA responses were not associated with M. bovis BCG vaccination status (OR 1.09 $(\mathrm{p}=0.66)$ and $0.81(\mathrm{p}=0.68)$ for blood and BAL, respectively) (fig. 1). However, the frequency of PPDresponsive BALMCs was higher in $M$. bovis BCG vaccinated versus unvaccinated persons, with a median (IQR) of 22 (6-101) versus 8 (1-44) spot-forming cells per 200000 BALMCs, respectively (marginal statistical significance, $\mathrm{p}=0.0468$ ), indicating a possible enhanced immune response in vaccinated subjects. With respect to $M$. tuberculosis-specific immune responses, though, no vaccination-related differences were found after stimulation with the antigens CFP-10 and ESAT-6. For CFP-10, a median (IQR) of 3 (0-17) versus 5 (1-15) spot-forming cells per 200000 BALMC was observed for vaccinated versus unvaccinated subjects, respectively; for ESAT-6, the median values (IQR) were 2 (0-19) versus 4 (0-11) spot-forming cells per 200000 BALMCs for the same respective groups. No difference in systemic or pulmonary M. tuberculosis-specific immune responses were observed among subgroups of HCWs and HHCs (data not shown).

We investigated the impact of M. bovis BCG vaccination during childhood on pulmonary and systemic M. tuberculosis-specific immune responses in healthy adult HCWs and HHCs with recent contact with tuberculosis patients. These immune responses are considered to be correlates of infection with M. tuberculosis [9]. Using our methodology, the results from this study suggest that vaccination has no substantial effect on the adaptive immune system with regards to protection against M. tuberculosis infection in adulthood.

The interpretation of the results is based on two observational and experimental findings. First, we did not observe a difference in the frequency of latent tuberculosis infection, assessed by IGRA responses from peripheral blood cells, among recent adult close tuberculosis contacts with and without a history of $M$. bovis BCG-vaccination in childhood. Second, local pulmonary ESAT-6- and CFP-10-specific immune responses measured by BAL-IGRA did not differ between the groups, while PPD-specific pulmonary immune responses were augmented in $M$. bovis BCG-vaccinated individuals. 


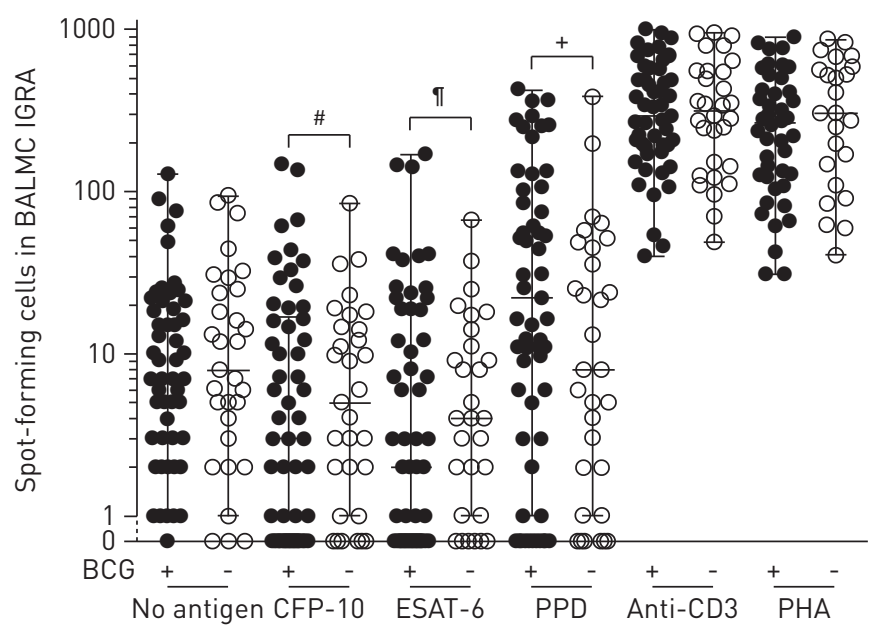

FIGURE 1 Numbers of spot-forming cells in the interferon (IFN)- $\gamma$ release assay (IGRA) (T-SPOT.TB) with bronchoalveolar lavage mononuclear cells (BALMCs). Mycobacterium bovis bacille Calmette-Guérin (BCG)-vaccinated subjects had a higher frequency of purified protein derivate (PPD)-responsive, IFN- $\gamma$-secreting cells in bronchoalveolar lavage compared with unvaccinated subjects. "No antigen" results are gross values. All other results are net values, i.e. the "no antigen" value has been deducted. Long horizontal lines indicate the median, short horizontal lines the interquartile range. The logarithmic scale allows the inclusion of all values into a single figure. CFP-10: 10-kDa culture filtrate protein; ESAT-6: 6-kDa early secretory antigenic target; PHA: phytohaemagglutinin. ${ }^{*}: \mathrm{p}=0.59 ;{ }^{\circ}: \mathrm{p}=0.94 ;{ }^{+}: \mathrm{p}=0.046$.

In summary, we present data from the largest cohort reported, to our knowledge, of healthy adult contacts of tuberculosis patients evaluated by bronchoscopy for pulmonary immune responses against M. tuberculosis and M. bovis BCG. In support of previous epidemiological data, we were unable to find a protective effect of $M$. bovis BCG vaccination against the development of latent infection with M. tuberculosis by adulthood [2]. The results underline the importance of the analysis of functional M. tuberculosis-specific immune responses from human lung cells for the assessment of correlates of immunity in future tuberculosis vaccine trials.

○ @ERSpublications

Childhood $M$. bovis BCG vaccination has no effect on protection against infection with M. tuberculosis in adults http://ow.ly/x09KU

Christian Herzmann ${ }^{1,2}$, Giovanni Sotgiu ${ }^{3}$, Tom Schaberg ${ }^{4}$, Martin Ernst ${ }^{5}$, Steffen Stenger ${ }^{6}$ and Christoph Lange ${ }^{1,7,8}$, for the German TB or Not TB Consortium ${ }^{9}$

${ }^{1}$ Division of Clinical Infectious Diseases, Research Center Borstel, Borstel, Germany. ${ }^{2}$ Center for Clinical Studies, Research Center Borstel, Borstel, Germany. ${ }^{3}$ Epidemiology and Medical Statistics Unit, Dept of Biomedical Sciences, University of Sassari, Research Medical Education and Professional Development Unit, AOU Sassari, Sassari, Italy. ${ }^{4}$ Center of Pneumology, Agaplesion Deaconess Hospital Rotenburg, Rotenburg, Germany. ${ }^{5}$ Division of Immune-Cell Analytics, Research Center Borstel, Borstel, Germany. ${ }^{6}$ Institute for Medical Microbiology and Hygiene, University Hospital of Ulm, Ulm, Germany. ${ }^{7}$ German Center for Infection Research (DZIF), Clinical Tuberculosis Unit, Borstel, Germany. ${ }^{8}$ Dept of Internal Medicine, University of Namibia School of Medicine, Windhoek, Namibia. ${ }^{9}$ For a list of the participating centres of the TB or Not TB network see the Acknowledgements section.

Correspondence: Christoph Lange, Division of Clinical Infectious Diseases, DZIF Clinical Tuberculosis Unit, Research Center Borstel, Parkallee 35, 23845 Borstel, Germany. E-mail: clange@fz-borstel.de

Received: March 042014 | Accepted after revision: May 142014 | First published online: June 252014

Support statement: This study was funded by the German Ministry of Education and Research (Bundesministerium für Bildung und Wissenschaft reference 01KI0784; grant to Christoph Lange, Steffen Stenger and Tom Schaberg). Christoph Lange is supported by the DZIF.

Conflict of interest: Disclosures can be found alongside the online version of this article at erj.ersjournals.com

Acknowledgements: The participating collaborators centres of the TB or Not TB network in Germany are: M. Allewelt, D. Anacker (both Evanglische Lungenklinik, Berlin), K. Avsar (Asklepios Fachkliniken München-Gauting, Munich), S. Blaas (Klinik Donaustauf, Donaustauf), J. Doehling (Research Center Borstel, Borstel), M. Fischer (Klinikum Nuremberg Nord, Nuremberg), A. Glaewe (Research Center Borstel, Borstel), P. Hammerl (Lungenfachklinik, Immenhausen), S. Jaletzky (Deakoness Hospital, Rotenburg), S. Junghanß (Fachkrankenhaus Coswig, Coswig), F. Kunitz (Helios Klinikum Emil von Behring, Berlin), L. Krabbe (Research Center Borstel, Borstel), S. Kohr (Agaplesion Pneumologische Klinik Waldhof, Elgershausen, Greifenstein), B. Lehnigk (Lungenclinic Großhansdorf, Großhansdorf), V. Leucht (Fachkrankenhaus Coswig, Coswig), J. Meywirth (Krankenhaus Diekholzen, Diekholzen), M. Mowe (Lungenklinik 
Hemer, Hemer), R. Muetterlein (Fachkrankenhaus Parsberg, Parsberg), A. Neher (Asklepios Fachkliniken MünchenGauting, Munich), M. Pletz (Hannover Medical School, Hannover), J. Paepke (Helios Klinikum Emil von Behring, Berlin), C. Prignitz (Krankenhaus Bethanien Solingen, Solingen), A. Quassem (Lungenfachklinik Lostau, Lostau), K.D. Schneider (Hannover Oststadtkrankenhaus, Hannover) and N. Vorreiter (Lungenfachklinik, Immenhausen).The authors are grateful for the technical assistance and support of Franziska Daduna, Jessica Hofmeister, Karoline Gaede, Stefan Niemann (all Research Center Borstel, Borstel, Germany), Daniel Mayer (Dept of Medical Microbiology and Hygiene, University of Ulm, Ulm, Germany), Jana Reppe, Lukas Tittmann (both Biobank Popgen, Kiel, Germany), and Steffi Jaletzki (Deaconess Hospital Rotenburg, Rotenburg, Germany).

\section{References}

Centers for Disease Control and Prevention. Global Routine Vaccination Coverage, 2011. MMWR Morb Mortal Wkly Rep 2012; 61: 883-885.

2 Abubakar I, Pimpin L, Ariti C, et al. Systematic review and meta-analysis of the current evidence on the duration of protection by bacillus Calmette-Guérin vaccination against tuberculosis. Health Technol Assess 2013; 17: 1-372. Faurholt-Jepsen D, Range N, PrayGod G, et al. BCG protects against tuberculosis irrespective of HIV status: a matched case-control study in Mwanza, Tanzania. Thorax 2012; 68: 288-289.

4 Basu Roy R, Whittaker E, Kampmann B. Current understanding of the immune response to tuberculosis in children. Curr Opin Infect Dis 2012; 25: 250-257.

5 Eisenhut M, Paranjothy S, Abubakar I, et al. BCG vaccination reduces risk of infection with Mycobacterium tuberculosis as detected by gamma interferon release assay. Vaccine 2009; 27: 6116-6120.

6 Abubakar I, Matthews T, Harmer D, et al. Assessing the effect of foreign travel and protection by BCG vaccination on the spread of tuberculosis in a low incidence country, United Kingdom, October 2008 to December 2009. Euro Surveill 2011; 16: 19526

7 Diel R, Loytved G, Nienhaus A, et al. Neue Empfehlungen fur die Umgebungsuntersuchungen bei Tuberkulose. [New recommendations for contact tracing in tuberculosis.]. Pneumologie 2011; 65: 359-378.

8 Haussinger K, Ballin A, Becker HD, et al. Empfehlungen zur Sicherung der Qualitat in der Bronchoskopie. [Recommendations for quality standards in bronchoscopy]. Pneumologie 2004; 58: 344-356.

9 Tameris M, Geldenhuys H, Luabeya AK, et al. The candidate TB vaccine, MVA85A, induces highly durable Th1 responses. PLoS One 2014; 9: e87340.

\section{Evaluation of Xpert MTB/RIF assay performance in diagnosing extrapulmonary tuberculosis among adults in a tertiary care centre in India}

\section{To the Editor:}

According to the World Health Organization Global Tuberculosis Report from 2013, there were 8.6 million incident tuberculosis (TB) cases globally and India alone contributed 26\% to this global scenario [1]. Of the five countries with the largest number of TB incident cases in 2012, India tops the list [1]. Epidemiological data suggest that extrapulmonary TB (EPTB) constitutes about 15-20\% of all TB cases, but among HIV-TB co-infection it accounts for 50\% of the cases [2]. Out of 1183373 new TB cases notified globally, 234029 (20\%) were reported to be cases of EPTB [1].

Difficulty in sampling from the extrapulmonary sites and the paucibacillary nature of the specimens make EPTB a diagnostic challenge. Dependency on smear microscopy in these samples may lead to higher false negative rates due to the low sensitivity of this technique. Mycobacterium tuberculosis (MTB) culture is quite a protracted technique, requiring well-trained laboratory personnel, and delay in diagnosis can cause more harm as the treatment is often started empirically.

Rapid nucleic acid amplification tests are emerging extensively to provide better yield for rapid diagnosis of TB. The Xpert MTB/RIF (Cepheid, Sunnyvale, CA, USA) is an automated, hemi-nested real-time PCR for detecting MTB complex and rifampin (RIF) resistance, which was initially evaluated for pulmonary specimens in large studies [3-5].

The present communication reports the performance of Xpert MTB/RIF in EPTB samples, with a large sample size from a single centre in a country with a high TB burden. 